\title{
Dielectric and AC conductivity behavior of Hexanoyl Chitosan-NaI based polymer electrolytes
}

\author{
F.H. Muhammad ${ }^{1}$, Asheila Jamal ${ }^{2}$, Tan Winie ${ }^{2, *}$ \\ ${ }^{1}$ Center of Foundation Studies, Universiti Teknologi MARA, 43800 Dengkil, Selangor, Malaysia \\ ${ }^{2}$ Faculty of Applied Sciences, Universiti Teknologi MARA, 40450 Shah Alam, Selangor, Malaysia
}

\section{A R T I C L E I N F O}

\section{Article history:}

Received 10 August 2016

Received in revised form

20 September 2016

Accepted 6 October 2016

\section{Keywords:}

Hexanoyl chitosan

Impedance spectroscopy

Dielectric properties

Electric modulus

\begin{abstract}
A B S T R A C T
Hexanoyl chitosan-sodium iodide (NaI) complexes were prepared using solution casting method. The temperature dependence conductivity was performed in the temperature range of 303-343K. From the dielectric studies, it has been observed that the dielectric constant, $\varepsilon_{r}$ and dielectric loss, $\varepsilon_{i}$ increases with temperature in the lower frequency region and almost negligible in the higher frequency region. This behavior can be explained on the basis of electrode polarization. Plot of real part, $M_{r}$ and imaginary part, $M_{i}$ of modulus versus frequency indicates that the systems are predominantly ionic conductor. Relaxation time calculated from the peak of $M_{i}$ at various temperatures appears to be thermally activated. The conductivity, $\sigma(\omega)$ is found to be almost frequency independent at low frequencies and became frequency dependent at high frequencies. The transference number measurement showed that the conductivity was predominantly ionic.
\end{abstract}

(C) 2016 The Authors. Published by IASE. This is an open access article under the CC BY-NC-ND license (http://creativecommons.org/licenses/by-nc-nd/4.0/).

\section{Introduction}

Solid polymer electrolytes (SPEs) are extensively studied due to their promising application in various electrochemical devices. The advantages of replacing conventional liquid electrolyte with SPE are ease of fabrication of films with desirable sizes, leakage-free, and wide electrochemical stability windows (Kuo et al., 2013; Ramesh et al., 2010; Winie and Shahril, 2015). The choice of a polymer to act as a host in a polymer electrolyte depends mainly on the presence of polar group with large sufficient electron donor to form coordination with cations of doping salt.

Chitosan, a natural polymer was chosen as a polymer host due to the presence of lone pair electrons at the nitrogen and oxygen atoms which enables coordination with the cation from the salt. Chitosan is attractive polymer host owing to their abundance sources, low cost and non-toxic (Aziz and Abidin, 2014; Leones et al., 2015). However, the insolubility of chitosan in a large number of solvents has limited it to be utilized widely. This is due to the rigid crystalline structure of chitosan caused by the

\footnotetext{
* Corresponding Author.

Email Address: tanwinie@salam.uitm.edu.my (T. Winie)

https://doi.org/10.21833/ijaas.2016.10.002

2313-626X/C) 2016 The Authors. Published by IASE

This is an open access article under the CC BY-NC-ND license

(http://creativecommons.org/licenses/by-nc-nd/4.0/)
}

formation of hydrogen bonding between amino and hydroxyl groups (Aziz et al., 2012).

Acyl modification of chitosan was carried out to improve the solubility of chitosan (Zong et al., 2000; Winie and Arof, 2004). In this study, hexanoyl substituted chitosan was prepared and it is known as hexanoyl chitosan. The salted hexanoyl chitosan electrolytes were prepared by incorporating $\mathrm{NaI}$ as the doping salt. NaI salt was chosen because of its availability in abundance at a cheaper cost. The softness of sodium metal promotes the stability of electrode-electrolytes interface in solid state ionic devices (Tripathi et al., 2012). The aim of this paper is to study the dielectric properties of SPE based on hexanoyl chitosan-NaI. The observed frequency and temperature dependence of ac conductivity were analyzed using existing theoretical models to investigate the conduction mechanism.

\section{Experimental}

Hexanoyl chitosan that exhibited solubility in tetrahydrofuran (THF) was prepared by acyl modification of chitosan. Chitosan with molecular weight of $6.0 \times 10^{5} \mathrm{~g} \mathrm{~mol}^{-1}$ from Aldrich was used in this work. Sodium iodide (NaI) with purity 99\% from Aldrich was used as received. The required amount of hexanoyl chitosan and different amount of $\mathrm{NaI}$ ranging from $5 \mathrm{wt}$. \% to $40 \mathrm{wt} \%$ were dissolved separately in THF in order to prepare hexanoyl chitosan-NaI system. The solutions were mixed 
together and stirred at room temperature for over 24 hours until complete dissolution. The homogeneous solutions were casted in Teflon petri dishes and allowed to evaporate slowly at room temperature to form films. The films were kept in a desiccator for continuous drying.

The impedance measurement of the films was carried out using HIOKI 3532-50 LCR Hi Tester in the frequency range of $50 \mathrm{~Hz}$ to $1 \mathrm{MHz}$ in the temperature range of $303-343 \mathrm{~K}$. The prepared electrolyte film was sandwiched between two stainless steel electrodes with diameter $1.2 \mathrm{~cm}$ under spring pressure. The ionic conductivity, $\sigma$ of the electrolytes sample was calculated using the equation:

$$
\sigma=t / R_{b} A
$$

where, $t$ is the thickness of the film and $A$ is the filmelectrode contact area. The bulk resistance, $R_{b}$ was determined from the complex impedance plot, $Z^{*}$ (Plot of imaginary, $Z_{i}$ versus real impedance, $Z_{r}$ ). The mathematical equation of impedance can be written as:

$$
Z^{*}=Z_{r}+j Z_{i}
$$

Using the impedance data, the dielectric constant, $\varepsilon^{*}$ and electrical modulus, $M^{*}$ were obtained using the following equations:

$$
\begin{aligned}
& \varepsilon_{r}(\omega)=Z_{i} / \omega C_{0}\left(Z_{r}^{2}+Z_{i}^{2}\right) \\
& \varepsilon_{i}(\omega)=Z_{r} / \omega C_{0}\left(Z_{r}^{2}+Z_{i}^{2}\right) \\
& M_{r}(\omega)=\varepsilon_{r} /\left(\varepsilon_{r}^{2}+\varepsilon_{i}^{2}\right) \\
& M_{i}(\omega)=\varepsilon_{i} /\left(\varepsilon_{r}^{2}+\varepsilon_{i}^{2}\right)
\end{aligned}
$$

Vacuum capacitance, $C_{0}=\varepsilon_{0} A / d, d$ is thickness of the electrolyte, $A$ is surface area of contact, $\varepsilon_{0}$ is vacuum space permittivity of $8.85 \times 10^{-12} \mathrm{~F} \mathrm{~m}^{-1}$ and $\omega$ is the angular frequency, being $2 \pi f$. $\varepsilon_{r}$ is the dielectric constant, $\varepsilon_{i}$ is the dielectric loss, $M_{r}$ is the real part of electric modulus and $M_{i}$ is the imaginary part of electric modulus, respectively.

The ionic transference has been determined by the DC polarization method using Solartron Analytical 1260 Impedance. The polarization current was monitored as a function of time on the application of a fixed dc voltage $(0.5 \mathrm{~V})$ across the sample sandwiched between two stainless steel electrodes.

\section{Result and discussion}

Dielectric constant $\varepsilon_{r}$ is a measure of a material's polarization which represents the stored electric charge. Since the charge is due to ions, increase in charge implies the increase in the number of ions (Winie and Arof, 2004). Dielectric loss, $\varepsilon_{i}$ is a measure of energy losses to move ions and align dipoles in every cycle of applied electric field and it is related to the electrical conductivity of materials (Tripathi et al., 2012).
The dependence of dielectric constant, $\varepsilon_{r}$ and dielectric loss, $\varepsilon_{i}$ as a function of frequency at various temperatures for hexanoyl chitosan-20wt.\% NaI system were plotted in Figs. 1(a) and (b). From Figs. 1 (a) and (b), the absence of relaxation peaks in both of the figures indicate that the increase in conductivity was primarily due to an increase in the number of free ions (Verma and Sahu, 2015). From Figs. 1(a) and (b), the values of $\varepsilon_{r}$ and $\varepsilon_{i}$ were rising sharply towards low-frequency region, which can be explained on the basis of electrode polarization, i.e. high charge accumulation at the electrodeelectrolyte interfaces.

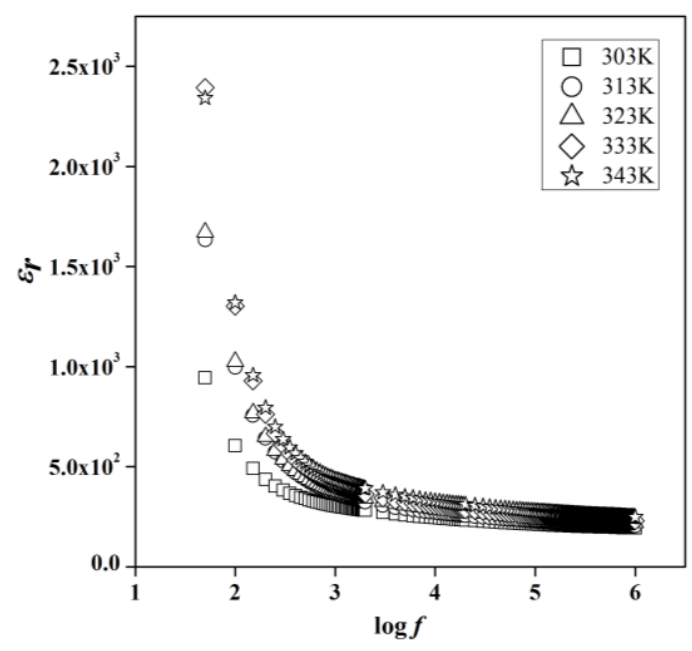

Fig. 1(a): Temperature dependent $\varepsilon_{r}$ vs. $\log f$ for hexanoyl chitosan-20wt.\% NaI

At low frequency, charge carriers get sufficient time to build up at the electrode-electrolyte interfaces before electric field was reversed hence contributes to the large electrode polarization. At high frequency, there was no time for the charge to build up at the electrode-electrolyte interface. It also can be seen from the figures that both $\varepsilon_{r}$ and $\varepsilon_{i}$ increase with temperature which can be contributed to the increase in the number of free ions with temperature. Temperature helps to dissociate salt into free ions.

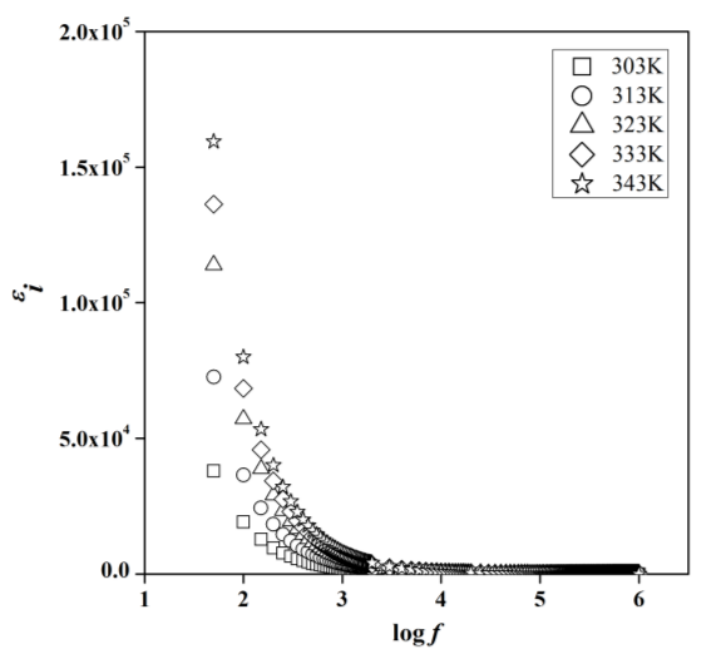

Fig. 1(b): Temperature dependent $\varepsilon_{i}$ vs. $\log f$ for hexanoyl chitosan-20wt. \% NaI 
Effect of electrode polarization can be suppressed using dielectric modulus analysis. The complex electric modulus has been used to investigate the conductivity relaxation phenomena. Figs. 2 (a) and (b) depict the real part, $M_{r}$ and imaginary part, $M_{i}$ of electrical modulus as a function of frequency at various temperatures for the same sample. It is clearly seen that $M_{r}$ increases with temperature in the high frequency region and approaches zero with the long tail at lower frequency. The long tail indicated the suppression of electrode polarization (Woo et al., 2012).

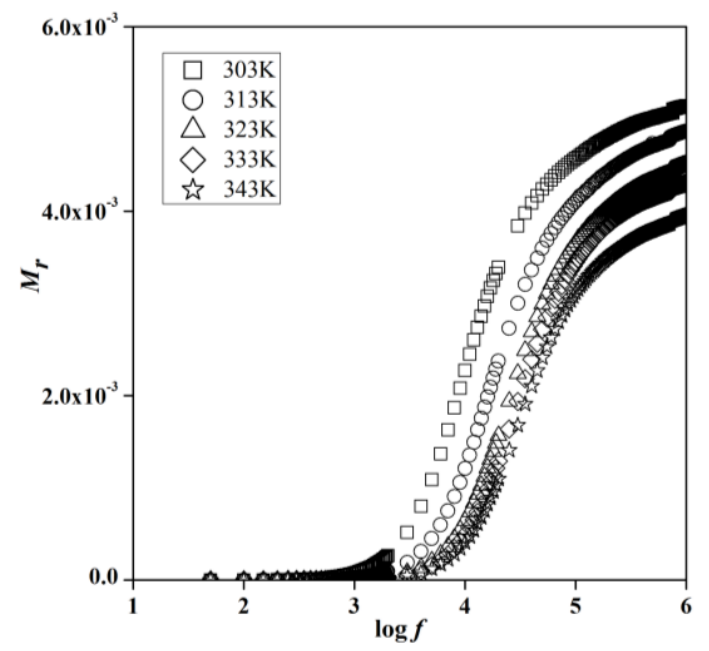

Fig. 2(a): Temperature dependence of $M_{r}$ vs. $\log f$ for hexanoyl chitosan-20wt.\% NaI

Fig. 2(b) shows the imaginary part, $M_{i}$ of electrical modulus. At high frequency region, the occurrence of peaks indicated that the electrolytes are ionic conductors. The broad and asymmetric peaks on the both sides of the maximum peak suggested the non-Debye behavior and can be understood as the results of distribution of relaxation time (Tripathi et al., 2012).

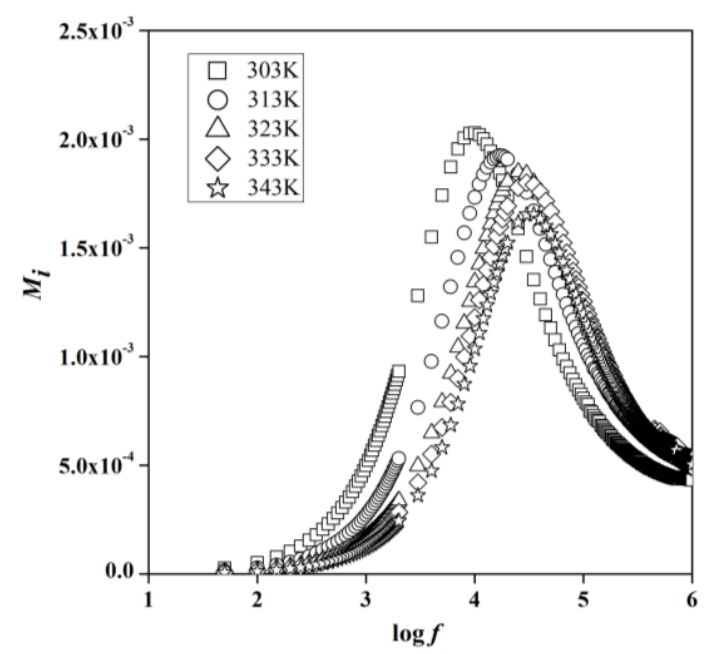

Fig. 2(b): Temperature dependence of $M_{i}$ vs. $\log f$ for hexanoyl chitosan-20wt.\% NaI

The angular frequency $\omega_{c}$ at which the maximum $M_{i}$ occurs represents the relaxation time, $\tau_{c}$ by: $\tau c \omega c=1$

The position of $\omega_{c}$ is found to shift towards the higher frequency as temperature increases. As temperature increases, the mobility of ions increases leads to a decrease in the relaxation time. The values of relaxation time and conductivity for hexanoyl chitosan-20wt.\% NaI at various temperatures are tabulated in Table 1 . These results suggested that the relaxation is a thermally activated process. As temperature increases, the relaxation time decrease which leads to increase in conductivity.

Table 1: The values of conductivity, $\sigma$ and relaxation time, $\tau_{c}$, for hexanoyl chitosan-20 wt\% $\mathrm{NaI}$ as a function of temperature

\begin{tabular}{|c|c|c|}
\hline Temperature,K & $\begin{array}{c}\text { Relaxation time, } \\
\tau_{c}(\mathrm{~s})\end{array}$ & $\begin{array}{c}\text { Conductivity, } \\
\sigma\left(\mathrm{Scm}^{-1}\right)\end{array}$ \\
\hline 303 & $2.0 \times 10^{-5}$ & $8.0 \times 10^{-7}$ \\
\hline 313 & $8.4 \times 10^{-6}$ & $1.9 \times 10^{-6}$ \\
\hline 323 & $6.4 \times 10^{-6}$ & $2.9 \times 10^{-6}$ \\
\hline 333 & $5.3 \times 10^{-6}$ & $3.1 \times 10^{-6}$ \\
\hline 343 & $4.6 \times 10^{-6}$ & $3.5 \times 10^{-6}$ \\
\hline
\end{tabular}

The frequency dependence of conductivity at different temperatures for hexanoyl chitosan20wt.\% NaI system were shown in Fig. 3. The conductivity is found to be almost frequency independent at low frequency region and became frequency dependent at the high frequency region. The frequency independent plateau represented the dc conductivity, $\sigma_{d c}$. It is observed that $\sigma_{d c}$ increases with temperature. The increase in $\sigma_{d c}$ with temperature is attributed to the increase in the number of free ions as support by Fig. 1(a) and the increase of ion mobility with temperature as support by Table 1 .

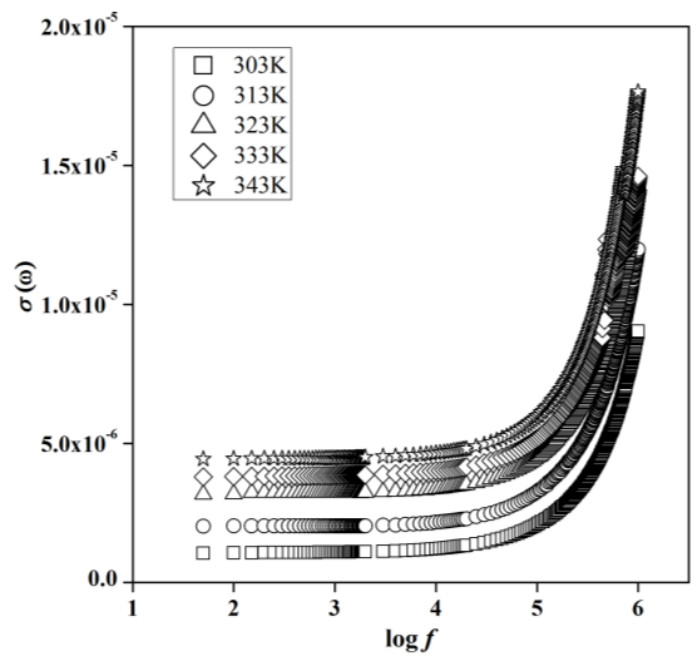

Fig. 3: Variation of AC conductivity with frequency for hexanoyl chitosan $-20 \mathrm{wt} . \% \mathrm{NaI}$ at different temperatures

The frequency dependence conductivity observed in Fig. 3 is in agreement with the prediction of the jump relaxation model (Funke, 1997). At lower frequencies, due to a longer period, an ion can jump to its neighboring vacant site successfully. Such successful jumps result in a long range translational 
motion of ions. Once an ion has arrived at the new complexation site, the ion may jump back to its initial site (backward jump) or it may relax at the new site before next forward jump. Probability of both backward jump and relaxation increases with frequency. This leads to the observed conductivity dispersion at the high frequencies (Funke and Wilmer, 2000).

The conductivity dispersion given by (Buraidah and Arof, 2011);

$$
\sigma_{a c}=A \omega^{s}=\varepsilon_{0} \varepsilon_{i} \omega
$$

thus, from Eq. 8,

$$
\varepsilon_{i}=\frac{A}{\varepsilon_{0}} \omega^{S-1}
$$

and

$$
\ln \varepsilon_{i}=\ln \frac{A}{\varepsilon_{0}}+(s-1) \ln \omega
$$

where, $A$ is a parameter dependent on temperature and $s$ is the power law exponent with value in the range between 0 and 1 . The value of $s$ can be obtained from the slope of $\ln \varepsilon_{i}$ versus $\ln \omega$ as shown in Fig. 4.

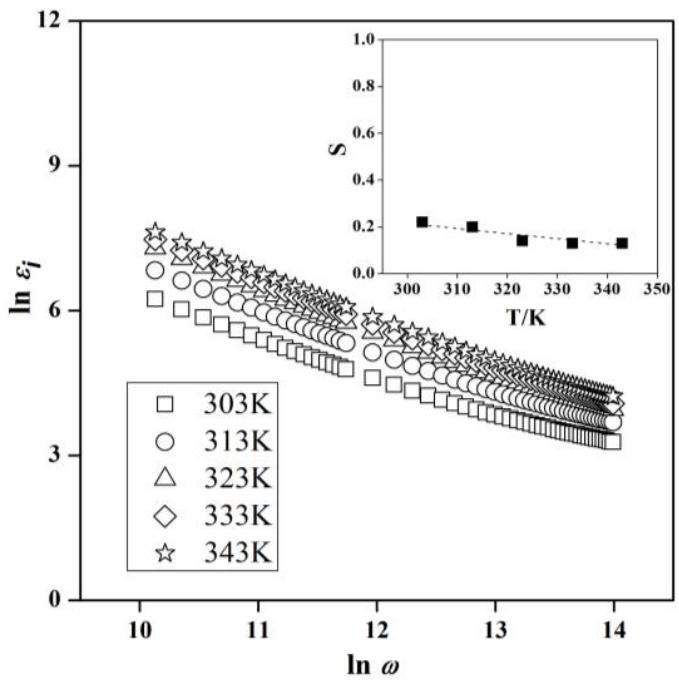

Fig. 4: $\ln \varepsilon_{i}$ versus $\ln \omega$ at different temperatures for hexanoyl chitosan-20wt.\% NaI.

According to the Jump relaxation model, the power law exponent $s$ are related to the rate of back jump to the site relaxation time as:

$s=$ back jump rate / site relaxation time

If $s<1$, probability of the ion to relax at the new site is greater than the probability of the ion to jump back to its initial site.

As shown in the insert of Fig. 4, the values of $s$ are in the range of 0.13 to 0.22 and it found to be temperature independent with a small gradient of 0.002. Different theoretical models have been proposed to correlate the temperature dependence of exponent, $s$ with the conduction mechanism of the materials. According to the quantum mechanical tunneling (QMT) model, the exponent $\mathrm{s}$ is almost temperature independent (Afifi et al., 2001). Thus the conduction mechanism for the present electrolyte system could be interpreted based on the QMT model. $\mathrm{Na}^{+}$ion jumps from one complexation site to another is accompanied by quantum mechanical tunneling (Psarras, 2006).

The ionic transference number for electrolyte containing 20 wt.\% NaI salt has been determined using DC polarization method. The current decays immediately and approaches steady state as shown in Fig. 5. The value of ionic transference number, $t_{i o n}$ was calculated from the plot of polarization current versus time using the equations,

$$
\begin{aligned}
& t_{\text {ion }}=\left(I_{i}-I_{f}\right) / I_{i} \\
& t_{e}=I_{f} / I_{i}
\end{aligned}
$$

Where, $I_{i}$ is the initial current and $I_{f}$ is the final current. The calculated value of ionic transference number is found to be 0.918 which suggest that the charge transport in the electrolytes system is predominantly due to ions.

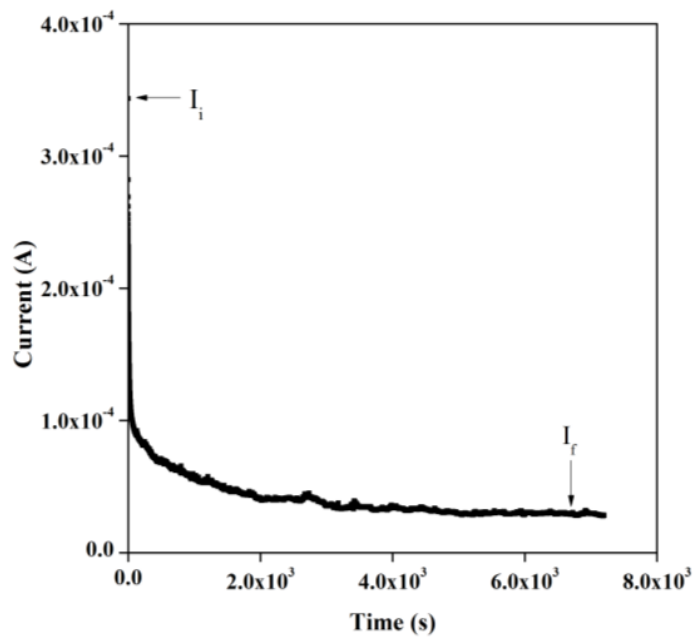

Fig. 5: Plot of transference number for hexanoyl chitosan20wt.\% NaI.

\section{Conclusion}

Studies on the dielectric behavior of hexanoyl chitosan-NaI complexes clearly showed that the values of $\varepsilon_{r}$ and $\varepsilon_{i}$ are strongly dependent on the frequency and temperature. The relaxation times for ionic charge carrriers were determined from the plot of $M_{i}$ vs. $\log f$. The conductivity, $\sigma(\omega)$ was found to obey the universal power law where it is almost frequency independent at low frequency region and frequency dependent at high frequency region. The QMT model was used to describe the conduction mechanism of present electrolyte system.

\section{Acknowledgement}

The authors wish to thank the Universiti Teknologi MARA and Ministry of Higher Education Malaysia for supporting this work through grant FRGS 5/3(7/2014) and DANA 5/3 REI (20/2015) 


\section{References}

Afifi MA, Bekheet AE, Elwahhab EA and Atyia HE (2001). Ac conductivity and dielectric properties of amorphous In 2 Se 3 films. Vacuum, 61(1): 917.

Aziz NA, Majid SR and Arof AK (2012). Synthesis and characterizations of phthaloyl chitosan-based polymer electrolytes. Journal of Non-Crystalline Solids, 358(12): 1581-1590.

Aziz SB and Abidin ZHZ (2014). Electrical and morphological analysis of chitosan: AgTf solid electrolyte. Materials Chemistry and Physics, 144(3): 280-286.

Buraidah MH and Arof AK (2011). Characterization of chitosan/PVA blended electrolyte doped with NH 4 I. Journal of Non-Crystalline Solids, 357(16): 3261-3266.

Funke K (1997). Ion transport in fast ion conductors-spectra and models. Solid State Ionics, 94(1): 27-33.

Funke K and Wilmer D (2000). Concept of mismatch and relaxation derived from conductivity spectra of solid electrolytes. Solid State Ionics, 136, 13291333.

Kuo C, Li W, Chen P, Liao J, Tseng C and Wu T (2013). Effect of plasticizer and lithium salt concentration in PMMA- based composite polymer electrolytes. International Journal of ELECTROCHEMICAL SCIENCE, 8: 5007-5021.

Leones R, Sentanin F, Nunes SC, Esperança JM, Gonçalves MC, Pawlicka A and Silva MM (2015). Effect of the alkyl chain length of the ionic liquid anion on polymer electrolytes properties. Electrochimica Acta, 184: 171-178.

Psarras GC (2006). Hopping conductivity in polymer matrix-metal particles composites. Composites Part A: Applied Science and Manufacturing, 37(10): 1545-1553.
Ramesh S, Liew CW, Morris E and Durairaj R (2010). Effect of PVC on ionic conductivity, crystallographic structural, morphological and thermal characterizations in PMMA-PVC blendbased polymer electrolytes. Thermochimica Acta, 511(1): 140-146.

Tripathi SK, Gupta A and Kumari M (2012). Studies on electrical conductivity and dielectric behaviour of PVdF-HFP-PMMA-NaI polymer blend electrolyte. Bulletin of Materials Science, 35(6): 969-975.

Tripathi SK, Jain A, Gupta A and Mishra M (2012). Electrical and electrochemical studies on magnesium ion-based polymer gel electrolytes. Journal of Solid State Electrochemistry, 16(5): 1799-1806.

Verma ML and Sahu HD (2015). Ionic conductivity and dielectric behavior of PEO-based silver ion conducting nanocomposite polymer electrolytes. Ionics, 21(12): 3223-3231.

Winie T and Arof AK (2004). Dielectric behaviour and AC conductivity of LiCF3SO3 doped $\mathrm{H}$ chitosan polymer films. Ionics, 10(3-4): 193-199.

Winie T and Shahril NSM (2015). Conductivity enhancement by controlled percolation of inorganic salt in multiphase hexanoyl chitosan/polystyrene polymer blends. Frontiers of Materials Science, 9(2): 132-140.

Woo HJ, Majid SR and Arof AK (2012). Dielectric properties and morphology of polymer electrolyte based on poly ( $\varepsilon$-caprolactone) and ammonium thiocyanate. Materials Chemistry and Physics, 134(2): 755-761.

Zong Z, Kimura Y, Takahashi $\mathrm{M}$ and Yamane $\mathrm{H}$ (2000). Characterization of chemical and solid state structures of acylated chitosans. Polymer, 41(3): 899-906. 